EPJ Web of Conferences 71, 00127 (2014)

DOI: $10.1051 /$ epjconf/20147100127

(C) Owned by the authors, published by EDP Sciences, 2014

\title{
The NICA Project at JINR Dubna
}

\author{
V.Kekelidze, A.Kovalenko, R.Lednicky, V.Matveev, I.Meshkov, A.Sorin, G.Trubnikov \\ Joint Institute for Nuclear Research, 141980 Dubna, Russia
}

\begin{abstract}
New scientific program is proposed at the Joint Institute for Nuclear Research (JINR) in Dubna aimed at study of hot and dense baryonic matter in the wide energy range from $2 \mathrm{GeV} / \mathrm{amu}$ to ${\sqrt{\mathrm{S}_{\mathrm{NN}}}}=11 \mathrm{GeV}$, and investigation of the nucleon spin structure with the maximum polarized protons and deuterons energy in the c.m. $27 \mathrm{GeV}$ (for protons). To implement this program the development of JINR's accelerator facility in high energy physics has started. This facility is based on the existing superconducting synchrotron - Nuclotron. The program foresees both experiments at beams extracted from the Nuclotron, and construction of an ion collider the Nuclotron-based Ion Collider fAcility (NICA).
\end{abstract}

\section{Introduction}

The new research facility NICA aimed at study of heavy ion and polarized proton and deuteron collisions is under design and construction at the Joint Institute for Nuclear Research (JINR) [1]. The study of hot and dense baryonic matter should shed light on: in-medium properties of hadrons and the nuclear matter equation of state (EOS); the onset of deconfinement (OD) and/or chiral symmetry restoration (CSR); phase transition (PT), mixed phase (see Figure 1 left) and the critical end-point (CEP); possible local parity violation in strong interactions (LPV) [2-5]. It is indicated in series of

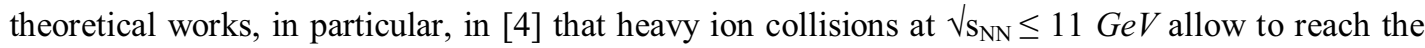
highest possible baryon density in the lab (Figure 1 right).
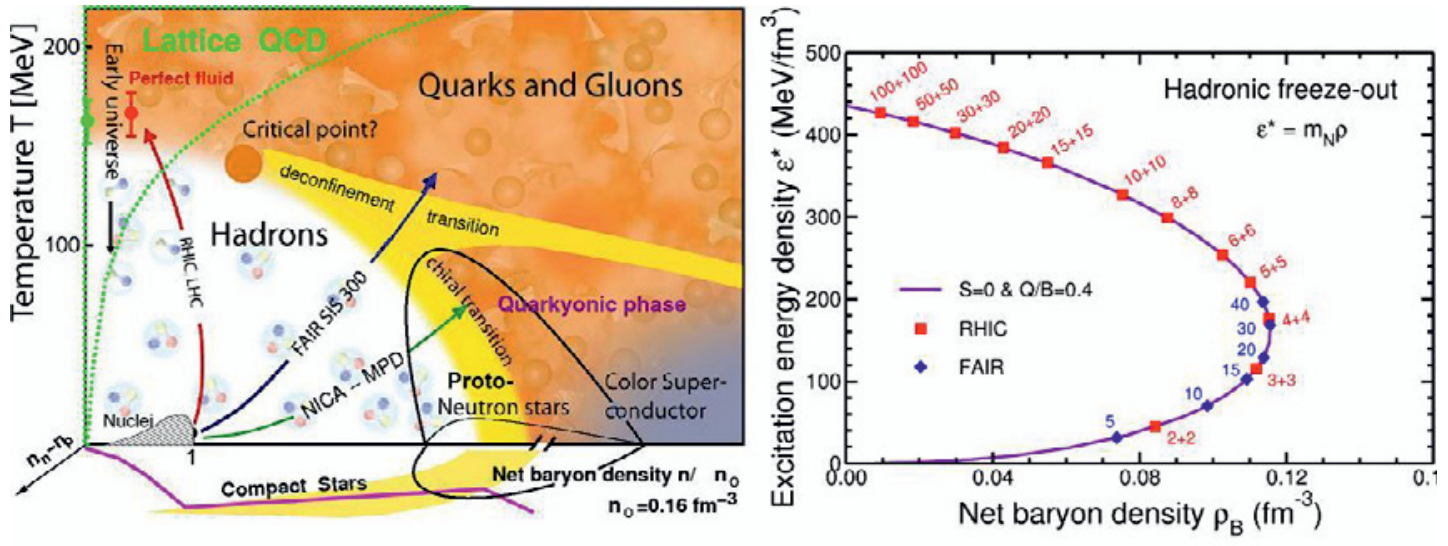

Figure 1. Left: phase diagram for QCD matter (mixed phase is indicated by yellow); right: freeze-out diagrams for baryonic matter indicating baryon density reachable at different energies in collider and fixed target experiments [4] (the region covered by the NICA experiments is indicated.

The high intensity and high polarization $(>50 \%)$ of colliding beams could provide a unique

This is an Open Access article distributed under the terms of the Creative Commons Attribution License 2.0, which permits unrestricted use, distribution, and reproduction in any medium, provided the original work is properly cited. 
possibilities for spin physics research, which is of crucial importance for the solution of the nucleon spin problem ("spin puzzle") - one of the main tasks of modern hadron physics.

The project NICA includes:

- upgrade of the existing superconducting synchrotron Nuclotron to provide a variety of ions up to Au with the maximum energy of $4.5 \mathrm{GeV} / \mathrm{u}$;

- the Baryonic Matter at Nuclotron (BM@N) experiment with ion beams extracted from the modernized Nuclotron;

- construction of the NICA collider providing collisions of various ions up to $A u^{79+}$ at the energy range up to $\sqrt{ }_{\mathrm{s}_{\mathrm{NN}}} \leq 11 \mathrm{GeV}$ with the luminosity $\mathrm{L}=1 \cdot 10^{27} \mathrm{~cm}^{-2} \mathrm{~s}^{-1}$, and polarized proton and deuteron beams up to c.m.s. energy of $27 \mathrm{GeV}$ for $p p$ collisions with the luminosity higher than $\mathrm{L}=1 \cdot 10^{32} \mathrm{~cm}^{-2} \mathrm{~s}^{-1}$ (at $\left.\sqrt{ } \mathrm{s}_{\mathrm{NN}}=27 \mathrm{GeV}\right)$ and different asymmetric collisions;

- an experiment with the MultiPurpose Detector (MPD) at the first (right-hand) interaction point (IP) of NICA with a primary goal to study heavy ion collisions;

- an experiment at the second (left-hand) IP of NICA with a primary goal to study spin physics.

The status of designed and operated heavy ion accelerators and colliders all over the world in the kinetic energy range from 1 to $50 \mathrm{GeV} / \mathrm{u}$ in lab system is as follows: 1) Dubna Nuclotron covered the region from 1 to $5.8 \mathrm{GeV} / \mathrm{u}$ for light ions $\left(\mathrm{d},{ }^{12} \mathrm{C}^{6+}\right)$ and from 1 to $1.5 \mathrm{GeV} / \mathrm{u}$ for heavy ions $\left({ }^{124} \mathrm{Xe}^{42+}\right)$. The next step in heavy ion acceleration at the Nuclotron is expected in March 2014 . The NICA collider will provide the collision parameters mentioned above; 2) The Brookhaven facility is capable to accelerate gold ions at AGS and at RHIC covering (theoretically) the energy range up to $11 \mathrm{GeV} / \mathrm{u}$ at a fixed target and to provide the desired $\sqrt{\mathrm{s}}_{\mathrm{NN}}$ from the targeted range, nevertheless reaching necessary luminosity ( $\mathrm{L} \sim 1 \cdot 10^{27} \mathrm{~cm}^{-2} \mathrm{~s}^{-1}$ ) at low energies is a problem; 3) SPS CERN mainly oriented at acceleration of lead ions. It is possible to perform fixed target experiments over the energy up to $158 \mathrm{GeV} / \mathrm{u}$ at the NA-49/61 set-up; 4) The new facility FAIR at GSI (Darmstadt, Germany) based on the construction heavy ion synchrotron SIS100 that should provide high intensity ion beams (up to uranium) for fixed target experiments over the energy range equivalent to $\sqrt{\mathrm{S}_{\mathrm{NN}}} \sim 3$ $6 \mathrm{GeV}$ with the further possible increase to $\sqrt{\mathrm{s}_{\mathrm{NN}}} \sim 5-8.6 \mathrm{GeV}$, when the SIS300 synchrotron would be put into operation. The last news from Japan gave evidence of the interest of Japanese physicists in heavy ion research, namely: the workshop on future development of the J-PARC facility aimed at heavy ions acceleration is scheduled for March 2014. Thus, it is clear - the research domain chosen for NICA at Dubna is important and attractive for the world scientific community. As one can see, the expected region of phase transition at the maximum baryon density corresponds to the NuclotronNICA capabilities.

\section{The NICA accelerator facility}

The Nuclotron is an existing synchrotron located at the Veksler and Baldin Laboratory of High Energy Physics (VBLHEP) of JINR, which was put into operation in 1993. It is based on the unique technology of superconducting fast cycling magnets developed at VBLHEP. The upgraded Nuclotron will provide proton, deuteron (including polarized) and multi charged ion beams. The magnetic field of dipole magnets $B=1.8 T$ corresponds to the ion beam energies: $5.2 \mathrm{GeV} / u$ for $\mathrm{d}(\mathrm{A}=2, \mathrm{Z}=1) ; 3.3$ 
$\mathrm{GeV} / u$ for $\mathrm{Xe}(\mathrm{A}=124, \mathrm{Z}=42)$; and $4.5 \mathrm{GeV} / u$ for $\mathrm{Au}(\mathrm{A}=197, \mathrm{Z}=79)$. The NICA facility (Figure 2) includes: an injection complex providing wide spectrum of ions up to the heaviest one Au at energy of $3.5 \mathrm{MeV} / u$ with an expected intensity of $2 \cdot 10^{9} \mathrm{ppc}$; a booster accelerating ions up to about

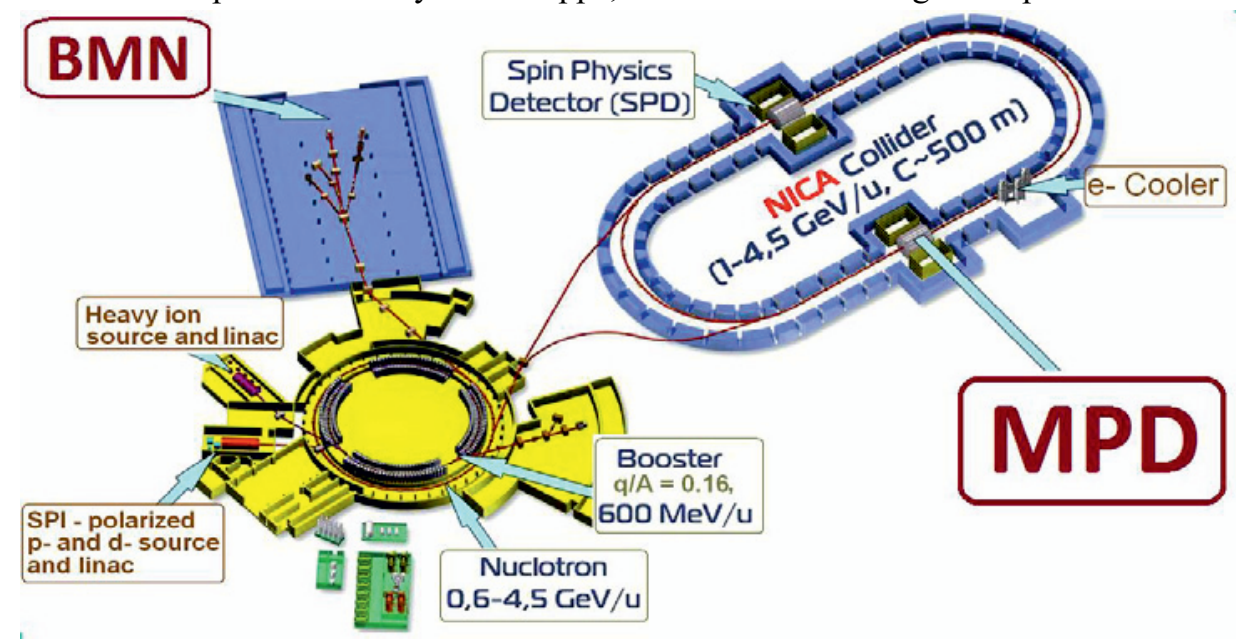

Figure 2. The new accelerator and experimental facility NICA.

$600 \mathrm{MeV} / \mathrm{u}$; the source of polarized ions (protons and deuterons) SPI with the linac accelerating light ions up to $5 \mathrm{MeV} / \mathrm{u}$; the Nuclotron continuing the acceleration up to maximum energy $4.5 \mathrm{GeV} / \mathrm{u}$ and two storage rings with two interaction points (IP). The ions are fully stripped before the injection into the Nuclotron. The major parameters of the NICA collider are the following: $B \rho_{\max }=45 \mathrm{Tm}$; vacuum in a beam chamber $-10^{-11}$ Torr; maximum dipole field $2 \mathrm{~T}$; ion kinetic energy range from $1 \mathrm{GeV} / u$ to $4.5 \mathrm{GeV} / u$ for $\mathrm{Au}^{79+}$; zero beam crossing angle at IP; $9 \mathrm{~m}$ space for detector allocations at IP's; average

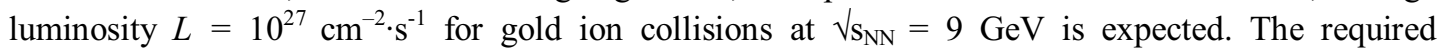
Nuclotron upgrade was started in 2008. Start to construction of the collider building and the MPD hall was done in December 2013 after the project passed the Russian State expertise (Figure 3).

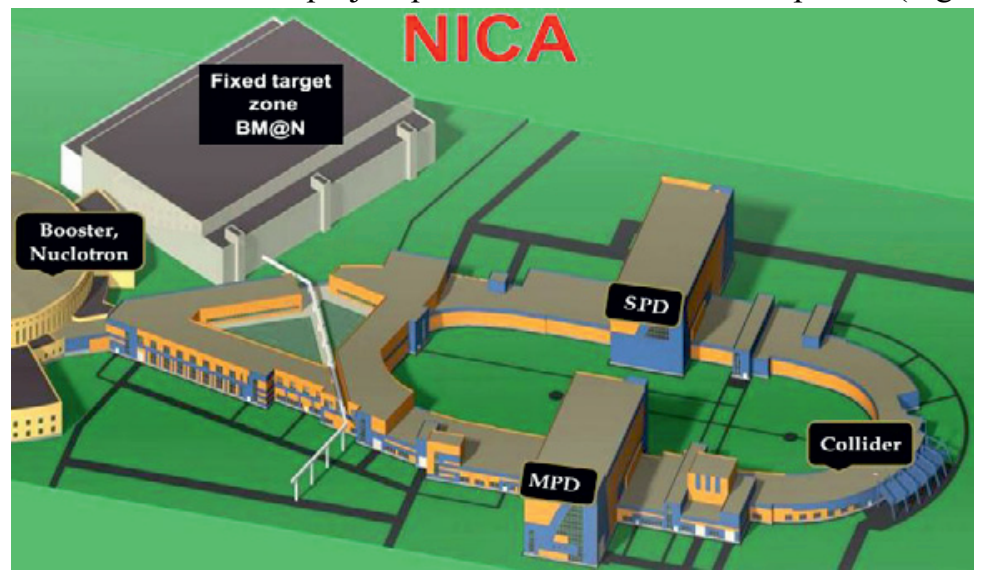

Figure 3. The architecture design of the NICA collider and experimental buildings at two IP's.

In the first (right-hand in respect to the beam injection direction) IP the Multipurpose Detector (MPD) will be installed, while a detector for the second IP is under design. A call for the corresponding proposal is announced. Work is in progress. 


\section{The BM@N experiment}

The energy of the extracted beams provided by the upgraded Nuclotron-NICA finally is reached to about $6 \mathrm{GeV} / \mathrm{u}$ for typical values of $\mathrm{A} / \mathrm{Z}=2$. Proton and deuteron beams could reach an intensity of $5 \cdot 10^{12}$ after construction of the booster ring will be completed and the accelerator will be put into operation. A typical variety of other possible beams and their intensities provided by Nuclotron-NICA are presented in Table 1.

Table 1: The Nuclotron-NICA beams.

\begin{tabular}{|l|l|l|l|l|l|l|l|}
\hline beam & ${ }^{7} \mathrm{Li}$ & ${ }^{12} \mathrm{C}$ & ${ }^{40} \mathrm{Ar}$ & ${ }^{56} \mathrm{Fe}$ & ${ }^{84} \mathrm{Kr}$ & ${ }^{124} \mathrm{Xe}$ & ${ }^{197} \mathrm{Au}$ \\
\hline p.p.p & $5 \cdot 10^{11}$ & $2 \cdot 10^{11}$ & $2 \cdot 10^{11}$ & $5 \cdot 10^{10}$ & $1 \cdot 10^{9}$ & $1 \cdot 10^{9}$ & $1 \cdot 10^{9}$ \\
\hline
\end{tabular}

The new fixed target set-up - BM@N (Baryonic Matter at Nuclotron) is under construction using the existing wide aperture dipole magnet, tracking chambers, time of flight (TOF) system, hadron calorimeter and a fast counter detector providing trigger signal. At the second stage the upgrade is foreseen to equip the set-up with a silicon vertex detector (in cooperation with GSI, Darmstadt) with an electromagnetic calorimeter and with a neutron detector (optional). The heavy-ion experiment $\mathrm{BM} @ \mathrm{~N}$ in the fixed-target hall of the Nuclotron will provide competitive research program focused on physics of dense nuclear and strange matter. The basic set-up (Figure 4, left part) shall comprise a large acceptance dipole magnet equipped with several highly granulated detector stations for track reconstruction. Additional time-of-flight detector provides particle identification. In order to measure rare diagnostic probes a high speed data readout and acquisition system is required for online event selection. Such a setup will be sufficient to perform a comprehensive study of strangeness production in heavy-ion collisions including multi-strange hyperons. Moreover, heavy-ion collisions in the Nuclotron energy range are very well suited for the production of (multi-strange) hypernuclei via the coalescence of lambda hyperons with light fragments. These measurements will provide new information on the properties of nuclear matter at neutron star core densities, and will open an avenue to explore the third (strangeness) axis of the nuclear chart. More detailed description of the physics case can be found at the NICA site. (http://www.nica.jinr.ru )

\section{The MPD program}

The MPD experimental program is aimed to investigate both hot and dense baryonic matter and polarization phenomena. Preliminary list of the first priority physics tasks to be performed includes: 1) measurement of a large variety of signals at systematically changing conditions of collision (energy, centrality, system size) using as bulk observables $4 \pi$ geometry particle yields (OD, EOS); multistrange hyperon yields and spectra (OD, EOS); electromagnetic probes (CSR, OD); azimuthal charged-particle correlations (LPV); event-by-event fluctuation in hadron productions (CEP); correlations involving $\pi, K, p, \Lambda$ (OD); directed and elliptic flows for identified hadron species (EOS,OD); reference data (i.e. $p+p$ ) will be taken at the same experimental conditions and 2) study of hyperon polarization and other polarization phenomena including possible study of the nucleon spin structure via the Drell-Yan (DY) processes after the MPD upgrade.

The MPD is a typical collider detector based on a superconducting solenoid with the magnetic field of $0.5 \mathrm{~T}(\sim 5 \mathrm{~m}$ in diameter and $\sim 8 \mathrm{~m}$ in length). It will be installed in the right-hand IP of the collider ring. The major sub-detectors of the MPD (Figure 4, right part) are: a time projection chamber (TPC); an inner tracker (IT); a time-of-flight (TOF) system; an electromagnetic calorimeter (ECal); an end cap tracker (ECT); two forward spectrometers based on toroid magnets (optional). There are foreseen three stages of putting the MPD into operation. The first stage involves the magnet, TPC, TOF, ECal (partially), IT (partially) start-up. At the second stage the end caps of the MPD will be fully equipped and some readout systems modernized. The third stage is related with forward spectrometers and is 
optional. The MPD experiment will be competitive and at the same time complementary to ones at RHIC [6], and FAIR [7].

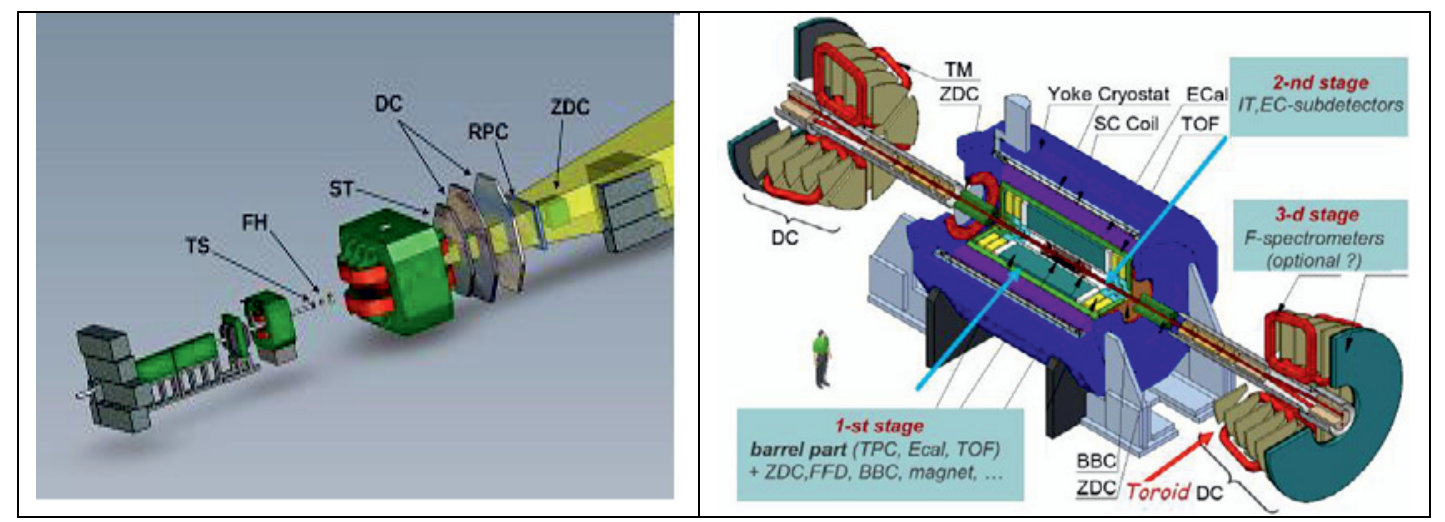

Figure 4. General view of the BM@N (left) and MPD setups.

The processes studied with the MPD were simulated using the dedicated software framework (MpdRoot). This software is based on the object - oriented framework FairRoot [8] and provides a powerful tool for detector performance studies, development of algorithms for reconstruction and physics analysis of the data. Evaluated rate in $\mathrm{Au}+\mathrm{Au}$ collisions at ${\sqrt{\mathrm{S}_{\mathrm{NN}}}}=7.1 \mathrm{GeV}(10 \%$ central interactions) taking into account the luminosity of $\mathrm{L}=1 \cdot 10^{27} \mathrm{~cm}^{-2} \mathrm{~s}^{-1}$ is $7 \mathrm{kHz}$. The corresponding particle yields are presented in Table 2 .

Table 2: The particle yields in $\mathrm{Au}+\mathrm{Au}$ collision (central) at ${\sqrt{\mathrm{S}_{\mathrm{NN}}}}=7.1 \mathrm{GeV}$.

\begin{tabular}{|l|l|l|l|l|l|}
\hline particle & yield $/ 4 \pi$ & yield at $\mathrm{y}=0$ & decay mode & $\varepsilon, \%$ & yield $/ 10 \mathrm{w}$ \\
\hline$\pi^{ \pm}$ & 290 & 100 & - & 61 & $2.6 \cdot 10^{11}$ \\
$\mathrm{~K}^{ \pm}$ & 60 & 20 & - & 60 & $4.3 \cdot 10^{10}$ \\
$\mathrm{p}$ & 140 & 40 & - & 60 & $1.2 \cdot 10^{11}$ \\
$\rho$ & 30 & 17 & $\mathrm{e}^{+} \mathrm{e}^{-}$ & 35 & $7.3 \cdot 10^{5}$ \\
$\omega$ & 20 & 10 & $\mathrm{e}^{+} \mathrm{e}^{-}$ & 35 & $7.2 \cdot 10^{5}$ \\
$\varphi$ & 2.6 & 1.2 & $\mathrm{e}^{+} \mathrm{e}^{-}$ & 35 & $1.7 \cdot 10^{5}$ \\
$\Omega^{-}$ & 0.14 & 0.1 & $\Lambda \mathrm{K}^{-}$ & 0.7 & $2.7 \cdot 0^{6}$ \\
\hline
\end{tabular}

More than ten working groups from 12 institutions are intensively working on the sub-detector $\mathrm{R} \& \mathrm{D}$ and on prototyping all detector elements. More detailed information could be found in the conceptual design report [9]. It was shown that the MPD is well optimized for studying in-medium effects caused by high baryon densities, such as: changing particle properties in hot and dense medium (broadening spectral functions etc.), event-by-event dynamical fluctuations of strange to non-strange particle ratios and others. These studies could be done with better precision than ones performed at the world's current experiments. The simulation of MPD capabilities shows that high statistics of studied events could be accumulated $\left(10^{9}\right.$ minimum bias events and $10^{8}$ central events per week), which provide the best precision for femtoscopy study with respect to correlation of multi-strange particles. In ten weeks of running more than $\sim 10^{6}$ of $\Omega$ - hyperon decays will be recorded.

Charged particles are reliably identified using both techniques: measuring $\mathrm{dE} / \mathrm{dx}$ of tracks in the TPC and by the TOF system (Figure 5). 

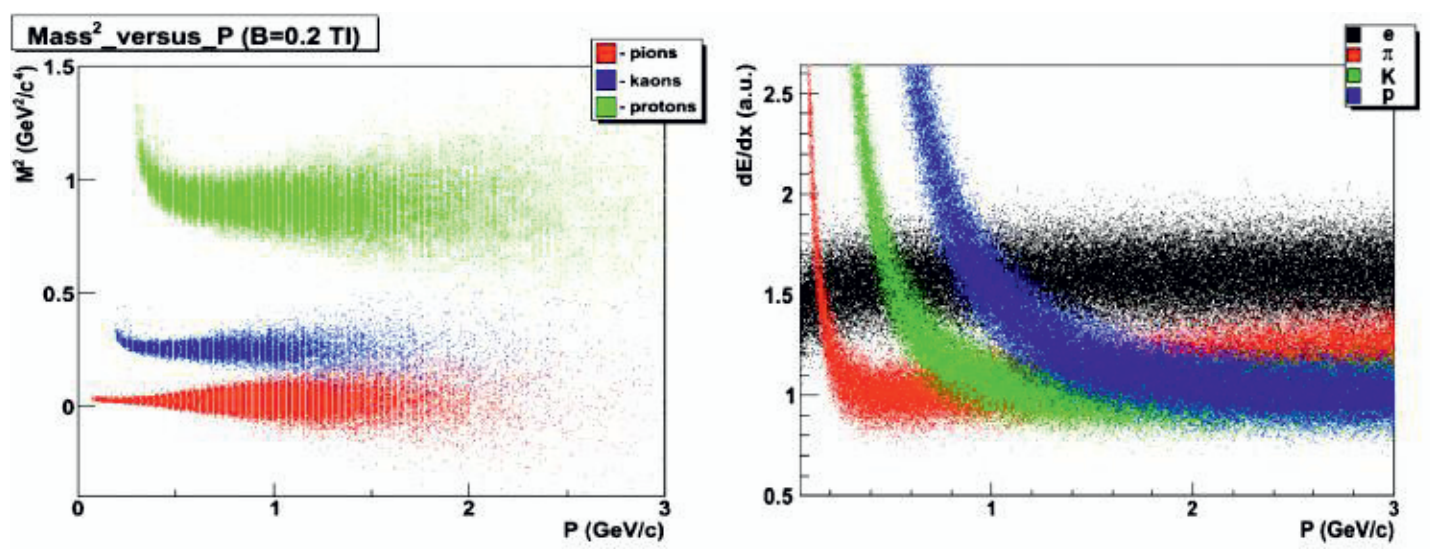

Figure 5. Left: particle ID using TOF system; right: particle ID in the TPC by measuring the losses due to the ionization.

There was obtained sufficiently high resolution of vertex reconstruction, for example $\Omega \rightarrow \Lambda \mathrm{K}^{-}$decay reconstruction implementing full chain of simulation: central $\mathrm{Au}+\mathrm{Au}$ collision generation at ${\sqrt{\mathrm{s}_{\mathrm{NN}}}}=$ $7.1 \mathrm{GeV}$ is at the level of $10 \mathrm{mkm}$. There are some novel proposals such as studying vorticity [10], helicity separation in heavy ion collisions [11] that could be studied at NICA. The MPD performance in general satisfies the required parameters for the proposed experimental program. The further optimization of MPD element design is continued. The corresponding infrastructure is developed as well at the site in the Veksler and Baldin Laboratory of High Energy Physics at JINR.

\section{Spin physics at NICA}

The NICA program foresees that the SPD detector will be designed and installed in the second IP. The physics program of the related experiment should be dedicated, first of all, to spin physics. There is a number of interesting processes which could be studied with this detector and with the fixed target detectors at beams extracted from the upgraded Nuclotron, namely: DY processes with longitudinally and transversally polarized $p$ and $d$ beams; extraction of unknown (poor known) parton distribution functions (PDF); PDFs from $J / \psi$ production processes; spin effects in various exclusive and inclusive reactions; cross sections of diffractive processes; helicity amplitudes and double spin asymmetries (Krisch effect) in elastic reactions; spectroscopy of quarkoniums with any available decay modes. This can be done in the kinematic region not available for other experiments. The formation of the collaboration has been started. The time scale of this experiment will be defined after consideration of the proposals. The important part of the NICA spin physics research program elaboration is adequate development of the infrastructure including the polarization control systems, polarimetery etc. The analysis of the accelerator problems connected with acceleration of polarized proton and deuteron beams was continued during the last three years. Feasible schemes of the polarization control in the Nuclotron and collider were proposed [12]. The last is particulary important because some of polarization phenomena experiments can be performed at the MPD set-up.

\section{Status of the construction}

The main part of R\&D work on the MPD detector and the new accelerators - NICA booster and collider was fulfilled mainly during 2009-2013. We have good conditions for starting mass production of the components. The list of the first order items is approved also. Preparation of the area for the collider and the detector buildings has been started (Figure 6). 


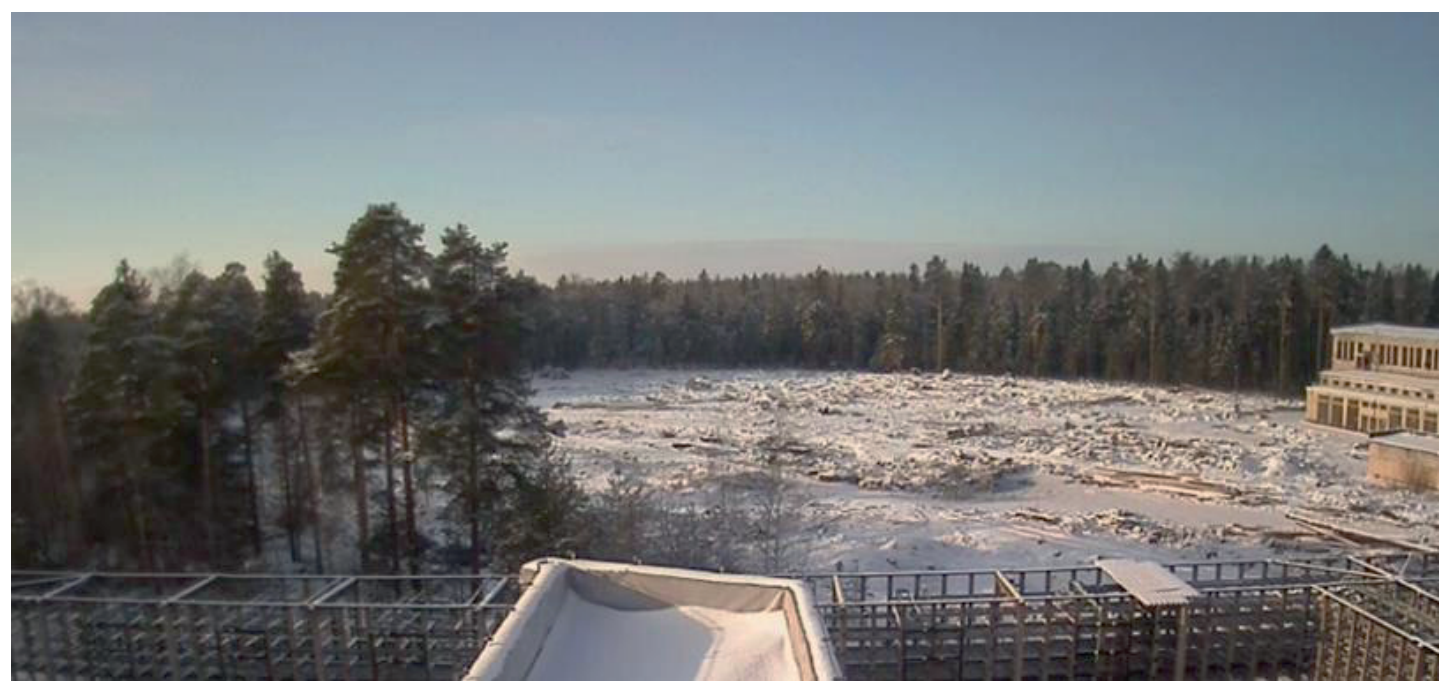

Figure 6. The area prepared for the NICA collider buildings.

\section{Conclusion and outlook}

The NICA accelerator and particle detector complex NICA to be constructed at VBLHEP JINR and the corresponding research program will provide relevant research which on one hand are competitive, and on the other hand are complementary to the ones being carried out and developing at other centers and facilities: RHIC at BNL, SPS and LHC at CERN, SIS18, SIS100 and SIS300 of FAIR at GSI site in Darmstadt. The spin physics at NICA has a good perspective to complement the experimental research at CERN SPS and, moreover, could give the unique possibilities of the experiments with polarized deuteron and proton collisions.

\section{References}

1. V.D.Kekelidze, A.D.Kovalenko, R.Lednicky, V.A.Matveev, I.N.Meshkov, A.S.Sorin, G.V.Trubnikov. PoS (ICHEP2012) 411, (2013).

2. A.Sissakian, A.Sorin, M.Suleimanov. V.Toneev, G.Zinovjev. Part.\& Nucl Lett. 5, 1 (2008).

3. M.Gazdzicki, M.Gorenstein, and S.Mrowczynski. PL., B585, 115 (2004).Mecke, I. Lee, J.R. Baker jr., M.M. Banaszak Holl, B.G. Orr, Eur. Phys. J. E 14, 7 (2004)

4. J. Cleymans, and J. Randrup, Phys. Rev. C74 (2006) 04791. M

5. B.Mohanty, J. Phys. G: Nucl.\& Part. Phys. 38 (2011) 124023.

6. G. S. F. Stephans, J. Phys. G32, S447 (2006), nucl-ex/0607030.

7. P. Senger. J. Phys. G30, S10S7 (2004).

8. D.Bertini et al., JoP,Conf.Ser.,119, 032011, (2008). http://cbmroot.gsi.de.

9. Multi Purpose Detector. CDR v.1.2 (JINR, Dubna, 2010); http://www.nica.ru

10. O.Rogachevsky, A.Sorin and O.Teryaev, PR, C, v82, (2010) 054910 [arXiv:1006.1331 [hep-ph]].

11. M.Baznat, K.Gudima, A.Sorin, O.Teryaev, PR, C, v.88, (2013) 061901[arXiv:1301.7003 [nu-th]].

12. A.D.Kovalenko, Y.N. Filatov, A.M.Kondratenko et al. Part.\&Nucl Lett. Vol.45, Issue 1 (2014). 